\title{
A Mitochondrial DNA Phylogeny Indicates Close Relationships between Populations of Lutzomyia whitmani (Diptera: Psychodidae, Phlebotominae) from the Rain-forest Regions of Amazônia and Northeast Brazil
}

\author{
EAY Ishikawa, PD Ready*/+, AA de Souza, JC Day*, EF Rangel**, \\ CR Davies***, JJ Shaw****
}

\begin{abstract}
Seção de Parasitologia, Instituto Evandro Chagas, Belém, PA, Brasil *Molecular Systematics Laboratory, Department of Entomology, The Natural History Museum, London SW7 5BD, UK **Departamento de Entomologia, Instituto Oswaldo Cruz, Rio de Janeiro, RJ, Brasil ***Department of Infectious and Tropical Diseases, London School of Hygiene \& Tropical Medicine, London, UK ****Departamento de Parasitologia, Instituto de Ciências Biomédicas, Universidade de São Paulo, São Paulo, SP, Brasil
\end{abstract}

Phylogenetic analysis of all 31 described mitochondrial (cytochrome b) haplotypes of Lutzomyia whitmani demonstrated that new material from the State of Rondônia, in southwest Amazônia, forms a clade within a lineage found only in the rain-forest regions of Brazil. This rain-forest lineage also contains two other clades of haplotypes, one from eastern Amazônia and one from the Atlantic forest zone of northeast Brazil (including the type locality of the species in Ilhéus, State of Bahia). These findings do not favour recognizing two allopatric cryptic species of $\mathrm{L}$. whitmani, one associated with the silvatic transmission of Leishmania shawi in southeast Amazonia and the other with the peridomestic transmission of Le. braziliensis in northeast Brazil. Instead, they suggest that there is (or has been in the recent past) a continuum of inter-breeding populations of $\mathrm{L}$. whitmani in the rain-forest regions of Brazil.

Key words: Lutzomyia whitmani - cryptic species - mitochondrial DNA lineages - cytochrome b - Leishmania transmission - Brazil

The phlebotomine sandfly Lutzomyia (Nyssomyia) whitmani (Antunes \& Coutinho, 1939) was first described from Ilhéus (IL), State of Bahia, Brazil and has a large range, mostly in Brazil but also extending into some neighbouring countries (Fig. 1, Young \& Duncan 1994). Rangel et al. (1996) recognized two phylogenetic lineages, which could be cryptic species: the form from the State of Pará (eastern Amazônia) transmits Leishmania shawi but is not noticeably synanthropic, unlike the typical form from northeast Brazil, which transmits Leishmania braziliensis and can be distinguished by the low abundance of a repetitive DNA sequence and, in the male, by a morphometric ratio - the length of genital filament: length of genital pump is greater. The Pará form was repre-

Support for fieldwork was received from the Fundação Nacional de Saúde, the Fundação Oswaldo Cruz, the Pronex programme of the Ministério de Ciências e Tecnologia, and the CNPq.

${ }^{+}$Corresponding author. Fax: +44-171-938. 8937. E-mail: P.Ready@nhm.ac.uk

Received 5 October 1998

Accepted 3 February 1999 sented by three populations [Fig. 1: Serra dos Carajás (CA), Dom Elizeu (DE), Monte Dourado, Jari (JA)], and two populations of the typical form were analysed (IL; Baturité, State of Ceará). Later, a phylogenetic analysis of mitochondrial (mt) DNA of Brazilian L. whitmani not only recognized the two regional lineages from Pará and the northeast but also discovered an additional lineage, the North-South, found mainly in the drier interior of Brazil [Fig. 1: Teresina (TS), Maringá (MA), Viana (VI)] (Ready et al. 1997), where L. whitmani is also implicated in the transmission of Le. braziliensis (Lainson et al. 1994).

The first study of mtDNA in L. whitmani included only one population from north of the River Amazon and no populations from western Amazônia (Ready et al. 1997). We can now fill some of the gaps in our knowledge of geographical variation in $L$. whitmani, following the recent collection of specimens from one location [NAV] in the State of Amapá, northeast Amazônia, and three locations [BRO, CAC, MON] in the State of Rondônia, southwest Amazônia (Fig. 1).

\section{MATERIALS AND METHODS}

Sandflies - The capture locations are given in Fig. 1. Specifically for the present study, specimens 
were collected from silvatic locations in Amapá [5 males, NAV, 1997] and Rondônia [4 males, BRO, 18 February 1998; 5 males and 5 females, CAC, 02 June 1998; and 4 males, MON, 21 October 1997]. Also, specimens from an older collection in Pará were characterized for the first time [ 4 males and 4 females, AP, 05 September 1994]. DNA sequences obtained from these sandflies were compared with those reported by Ready et al. (1997), who gave information about the specimens they used from silvatic locations in Pará [AP, CA, DE, JA], and from peridomestic locations in the states of Pernambuco [RE], Bahia [CP, IL], Piauí [TE], Paraná [MA] and Espírito Santo [VI]. Specimens from DE, JA, and IL were $\mathrm{F} 1$ progeny reared in the insectary of the Instituto Evandro Chagas, Belém, while all other specimens were wild-caught, usually in miniature CDC light-traps or by aspirating from tree bases, Shannon traps or domestic animal shelters (Ready et al. 1983). Sandflies were killed by freezing or with cigarette smoke, and then stored either in analytical grade $96 \%$ ethanol at $4 \%-20^{\circ} \mathrm{C}$ [specimens from locations NAV, BRO, CAC, MON, AP (1995), CP] or in liquid nitrogen [AP (1994), the rest] before being taken to London on dry ice. Identification of $L$. whitmani was based on external characters (male genitalia, infuscation patterns, relative lengths of palpal segments) and on the morphology of female spermathecae and cibaria viewed in abdominal terminalia and heads slide-mounted in Berlese fluid (Young \& Duncan 1994).

Extraction and amplification of DNA - Total genomic DNA was extracted from frozen single sandflies by the methods of Ready et al. (1997). Specimens stored in ethanol were washed twice in 'Analar' grade water (BDH/Merck), soaked in the same overnight, and then blotted dry before processing. Usually with these methods, 50-250 ng of DNA is extracted from each sandfly and, following ethanol precipitation, the genomic DNA was dissolved in $20 \mathrm{ml}$ of $1 \mathrm{x}$ TE (10mM Tris. $\mathrm{HCl}, 1 \mathrm{mM}$ EDTA pH 8.0). 0.2-2 $\mathrm{ml}$ of this DNA solution was added to each $50 \mathrm{ml}$ of polymerase chain reaction (PCR) mix, which contained Promega buffer (without $\mathrm{MgCl}_{2}$ ), $1.5 \mathrm{mM} \mathrm{MgCl} 2,60 \mathrm{mM}$ of each dNTP, and $100-500 \mathrm{ng}$ of each primer. The forward primer CB3-PDR (5'-3' CA(T/C)ATTCAACC(A/ T)GAATGATA) and the reverse primer N1N-PDR (5'-3' GGTA(C/T)(A/T)TTGCCTCGA(T/A)TTCG (T/A)TATGA) were used to amplify a 544 basepair (bp) fragment of mtDNA, which contains the 3 ' end of the cytochrome b gene (Ready et al. 1997). The primer sequences are homologous to nucleotides 11214-11233 and 11759-11734, respectively, of the mitochondrial genome of Anopheles gambiae (Beard et al. 1993). PCR was performed in an Hybaid Omnigene thermal cycler set for: an initial denaturation step at $94^{\circ} \mathrm{C}$ for $3 \mathrm{~min}$; a hold at $80^{\circ} \mathrm{C}$, when 1.5 units of Promega Taq Polymerase was added for a "hot start"; 5 cycles of $94^{\circ} \mathrm{C}$ for $30 \mathrm{sec}, 38^{\circ} \mathrm{C}$ for $30 \mathrm{sec}$ and $72^{\circ} \mathrm{C}$ for $1.5 \mathrm{~min}$; 35 cycles of $94^{\circ} \mathrm{C}$ for $30 \mathrm{sec}, 42^{\circ} \mathrm{C}$ for $30 \mathrm{sec}$ and $72^{\circ} \mathrm{C}$ for $1.5 \mathrm{~min}$; a final extension period of 10 min at $72^{\circ} \mathrm{C}$; and cooling to room temperature. At the end of the reaction, the overlaying mineral oil was removed with chloroform and the PCR products purified by fractionation in $1 \%$ agarose gels followed by binding to glassmilk (Geneclean II, BIO 101 Inc.).

DNA sequencing of PCR products - The DNA purified from each PCR product was redissolved in 5-15 ml of 'Analar' water (BDH/Merck) to give a concentration of ca. 15-20 ng/ml. Fluorescent cycle sequencing was performed with 50-100 ng of this double-stranded DNA in each $20 \mathrm{ml}$ sequencing reaction mix, prepared using the $A B I$ PRISM $^{\text {TM }}$ dRhodamine Cycle Sequencing Ready Reaction Kit (Applied Biosystems Inc.) and 3.2 pmoles of one of the PCR primers. A Techne "Genius" thermal cycler was set for: an initial denaturation at $40^{\circ} \mathrm{C}$ for $2 \mathrm{~min}, 94^{\circ} \mathrm{C}$ for $2 \mathrm{~min}$; followed by 35 cycles of $96^{\circ} \mathrm{C}$ for $15 \mathrm{sec}, 50^{\circ} \mathrm{C}$ for $15 \mathrm{sec}$ and $60^{\circ} \mathrm{C}$ for $4 \mathrm{~min}$; and, after completion, a hold at $4^{\circ} \mathrm{C}$ (ABI protocol, with modifications). Upon completion of the cycle sequencing reactions, the extension products were purified (by ethanol precipitation, method 1) and prepared for polyacrylamide gel electrophoresis, and the DNA sequences were read using the ABI 373A or 377 systems, all according to $\mathrm{ABI}$ protocols.

DNA sequence analysis - DNA sequences were edited and aligned using SeqEd version 1.0.3 software $(\mathrm{ABI})$, and phylogenetic analyses were performed with PAUP version 3.1.1 software (Swofford 1993).

\section{RESULTS}

Diversity and distribution of mitochondrial haplotypes - New mtDNA sequences were obtained from 31 adult $L$. whitmani, all from Amazônia, and aligned with the 18 unique 450-bp sequences of this species previously reported by Ready et al. (1997) (mtDNA haplotypes whit1-18; consensus sequence: GenBank accession no. U80966). These sequences consist of the 3 ' ends of the cytochrome $b$ (cyt b) and NADH1 genes together with the intervening tRNAser ${ }^{\mathrm{TCN}}$ gene and intergenic spacers. All of the informative substitutions (i.e. DNAbase character-state changes) were found in the last 294 nucleotides of cyt b, which were analysed by deriving data and distance matrices with PAUP (Swofford 1993). Only 3 out of the 31 new DNA sequences belonged to previously known cyt $\mathrm{b}$ haplotypes, and these came from L. whitmani 
caught in Pará, eastern Amazônia (Fig. 1): 2 AP sequences were identical to haplotypes whit15/16 (from locations AP and DE) and 1 AP sequence was identical to haplotype whit17 (from location CA) (some of the 450-bp haplotypes do not differ in their cyt $b$ sequences, and therefore their numbers are united). However, the other 28 new sequences belonged to 13 previously unreported cyt b haplotypes: 4 haplotypes (whit 19-22) came only from AP, 1 haplotype (whit23) from both AP and NAV (northeast Amazônia), 1 haplotype (whit24) only from NAV, and 7 haplotypes (whit25-31) only from Rondônia (locations BRO, CAC, MON, in southwest Amazônia) (Figs 1, 2).

Phylogenetic relationships among mitochondrial haplotypes - The new 294-bp cyt b haplotypes did not fall into discrete groups based on uncorrected pairwise sequence dissimilarity (or genetic distance): AP haplotypes differed from each other by only $0.3-1 \%$, similar to the $0.3-1.4 \%$ range for the whole of eastern Amazônia (locations AP, CA, DE, JA and NAV); and, CAC haplotypes differed from each other by $0.3-2.4 \%$, which is the range for all three locations in Rondônia (BRO, CAC, $\mathrm{MON}$ ) and overlaps the 1.0-3.4\% range of pairwise sequence dissimilarities between the haplotypes of eastern Amazônia and Rondônia. Therefore, the sequence data were analysed phylogenetically, in order to resolve relationships among the haplotypes.

A maximum parsimony analysis was carried out on the 294-bp alignment of all 31 cyt b haplotypes of L. whitmani by using PAUP (Swofford 1993). A single shortest tree of 38 steps was found during 100 heuristic searches (each beginning from a starting tree acquired with a random-addition sequence and TBR branch swapping), when all characters were unordered and weighted equally, and the haplotypes of the NorthSouth lineage were used as an outgroup to root the tree (Fig. 3). The level of support for each branch was estimated using bootstrap analysis within PAUP (100 replicates, each with 10 heuristic searches using random addition and TBR branch swapping) and the Bremer index, i.e. the number of tree steps that can be added before a branch is

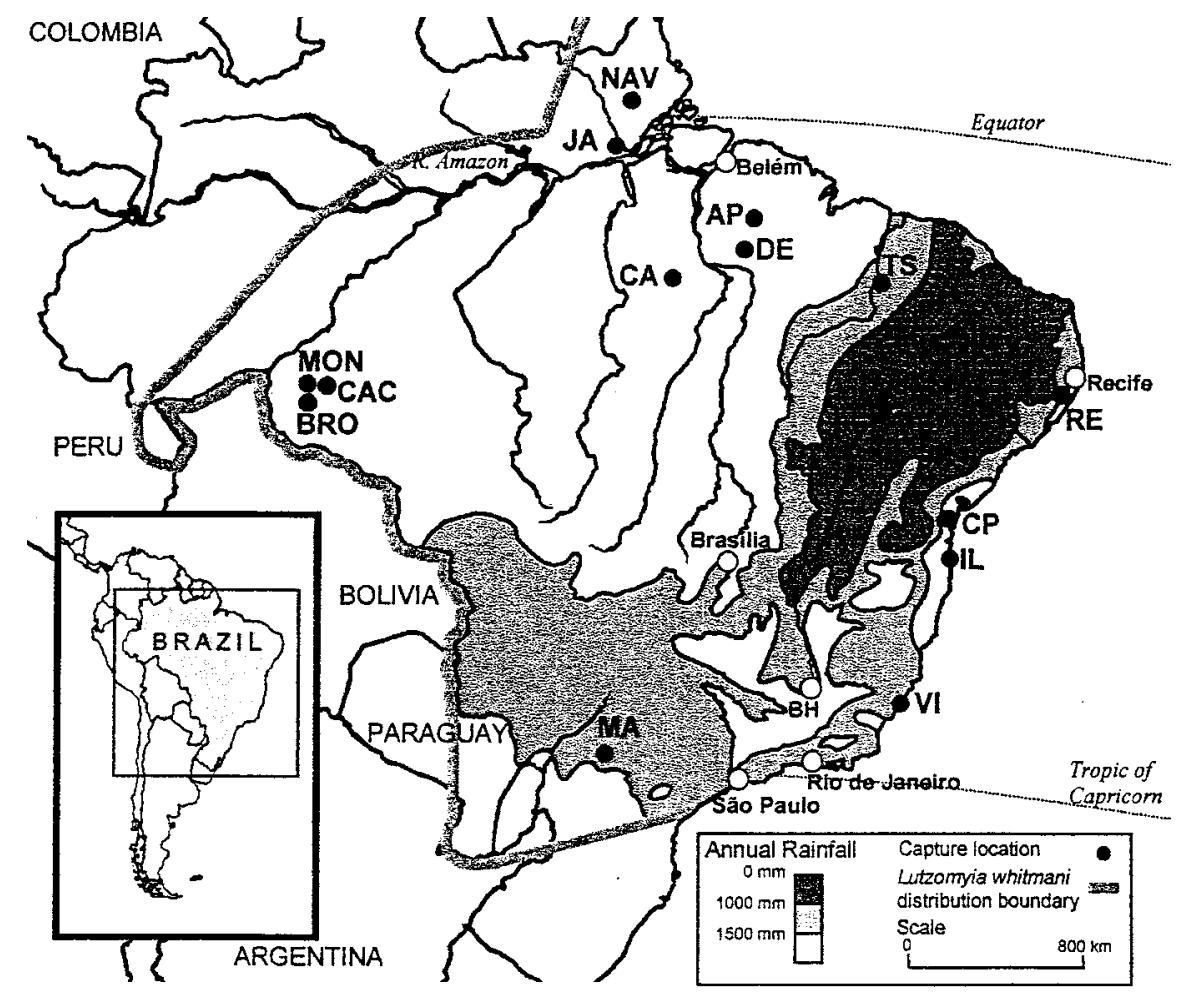

Fig. 1: capture locations of Lutzomyia whitmani related to annual rainfall (Haffer 1987) within its range (Young \& Duncan 1994). Capture locations - State of Amapá: Serra do Navio (NAV); State of Bahia: Corte de Pedra (CP), Ilhéus (IL); State of Espírito Santo: Viana (VI); State of Pará: Água Parada near Paragominas (AP), Serra dos Carajás (CA), Dom Elizeu (DE), Monte Dourado (JA); State of Paraná: Maringá (MA); State of Pernambuco: Refrigério near Amaraji (RE); State of Piauí: Teresina (TS); State of Rondônia: Buriti (BRO), Cacaulândia (CAC), Montenegro (MON). 


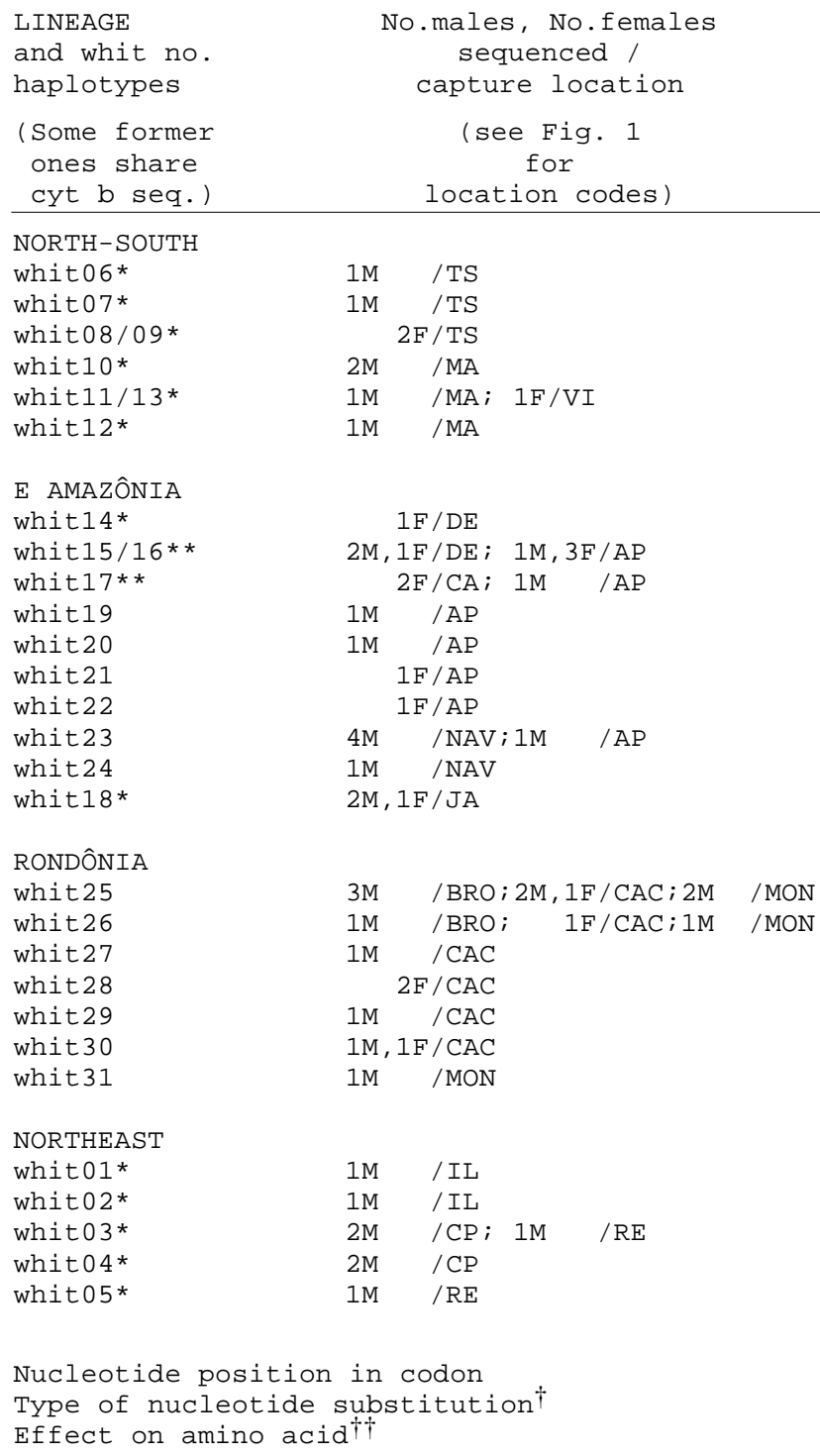

Position of the 26 variant characters in last 294 bp of cyt b (positions 3-288)

1111111122222222222

2466790113899901345777888 34509892458002510149038678

GAGACAGCAACGGTTTACAGTAATCA AAGACAGCAACGGTTTACAGTGA $\underline{\underline{T C A}}$ AAGACAGCAACGGTTTACAGTAATCA AAAACAACAACAGTTTACAGTAATCA AAAACAACAACAGTTTACAGTAGTCA AAAACAACGACAGTTTACAGTAGTCA $\pm$

AGAATAACAACAGCTTACAGTAGGCT AGAATAACAACAGTTTACAGTAGGCT AGAATAATAACAGTTTACAGTAG $\underline{\underline{G C T}}$ AAAATAGCAACAGTTTACAATAGGCT AGAATAACAACAGTTTTCAGTAGGCT AAAATAACAACAGTTTACAGTAGGCT AGAATAACAGCAGTTTACAGTAG $\underline{\underline{G C T}}$ AAAATAGCAACAGTTTACAGTAGGCT AAAATAGCAACTGTTTACAGTAGGCT AAAATAGCAACAGTTTACAGTAGGAT

AGAATAACAACAGTTCATAGTAAGCA AGAATGACAACAGTTCATAGTAA $\underline{\underline{G C A}}$ AGAATAATAATGGTTCACCGTAAGCA AAAATAACAACAGTTCATAGTAAGCA AGAATAACAACGGTTCATAGTAAGCA AAAATAACAACAGTTCATAGTAAGCT AAAATAGCAACAGTTCACAGTAAGCA $\pm \quad \pm$

AAAGCAACAACGGCCCACAGTAGGCA AAAGCAACAACGACCCACAGTAGGCA AAAGTAACAACGGCCCACAGTAGGCA AAAGTAACAACGGCCCACAGCAGGCA AAAATAACAACGGCCCACAGTAGGCA $\pm \pm+ \pm$

33333333333313333311332123 iiiiiiiiibiiiviviiivvv SSSSSSSSNSSNSSSSSNNSSNNNS

Fig.2: cytochrome b haplotypes of adult males (M) and females (F) of Lutzomyia whitmani from different Brazilian locations, grouped by geographical lineage and characterized by variant 3' nucleotides. Haplotypes were found: *only in a previous study (Ready et al. 1997), **in previous and present studies, or only in the present study (the rest). Lineage synapomorphies are: $+=$ diagnostic, $\pm=$ informative. Penultimate codon is underlined. ${ }^{\dagger}$ Nucleotide substitutions are transitions (i: $A<->\mathrm{G}, \mathrm{C}<->\mathrm{T}$ ), transversions (v: A<->C, A<->T, G<->C, G<->T) or both (b). ${ }^{\dagger}$ Substitutions are non-synonymous (N, with amino acid replacement) or synonymous (s, without amino acid replacement).

lost in the consensus of near-most-parsimonious trees (Bremer 1994). We conclude from our maximum parsimony analysis that the cyt $b$ haplotypes from eastern Amazônia form a monophyletic group, which is the sister clade to those from Rondônia and the northeast (Fig. 3). All three clades form a well supported in-group (diagnostic character 286, bootstrap $>50 \%$, Bremer index $=$ 1 ), but within this only the northeast clade is well supported (diagnostic character 195, bootstrap $>50 \%$, Bremer index $=1)($ Figs 2, 3).
The haplotypes of the North-South lineage were chosen as an outgroup for the following reasons: phylogenetic analyses of DNA sequences of the nuclear small-subunit ribosomal RNA gene and of cyt $b$ have shown that the North-South lineage forms the first branch within L. whitmani when analysing a range of species from Nyssomyia and other phlebotomine subgenera (PD Ready, E Galati, JC Day \& AA de Souza, unpublished observations); relationships among the remaining mtDNA lineages of $L$. whitmani are not unambiguously 


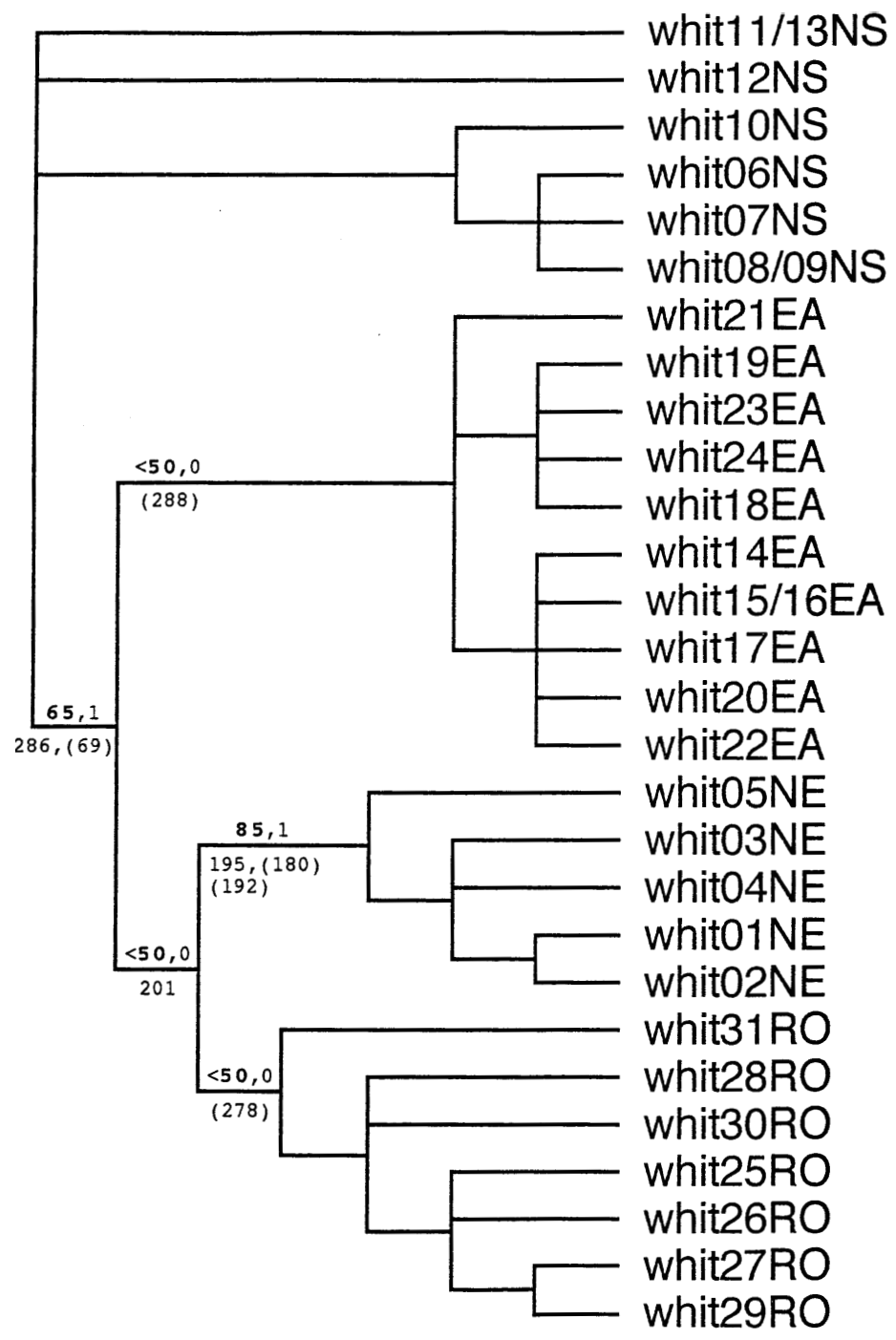

Fig. 3: phylogenetic tree (cladogram) of mitochondrial cytochrome b haplotypes of Lutzomyia whitmani (whit1-31) obtained by maximum parsimony analysis (PAUP: Swofford 1993). A single shortest tree (38 steps, rescaled consistency index $=0.62$ ) was found during 100 heuristic searches (random-addition sequence and TBR branch swapping), when all characters were unordered and weighted equally, and the NS haplotypes were used as an outgroup to root the tree. Geographical haplotype lineages: NS = North-South; EA = eastern Amazônia; $\mathrm{NE}$ = Northeast; RO = Rondônia. Numbers above each branch: bold = \% bootstrap support for the node (100 replicates, each with 10 heuristic searches); other = Bremer index, i.e. the number of tree steps that can be added before a branch is lost in the consensus of near-most-parsimonious trees (Bremer 1994). Numbers below each branch: diagnostic and (other informative) synapomorphic characters.

resolved by using cyt b haplotypes of other Nyssomyia species as an outgroup, because the greater genetic distances between species demand the use of differential character weighting (PD Ready, E Galati, JC Day \& AA de Souza, unpublished observations); and, it is no longer possible to follow Ready et al. (1997) and select as the outgroup the cyt b haplotypes of Lutzomyia (Nyssomyia) intermedia (Lutz \& Neiva, 1912), because now there is known to be mitochondrial introgression between L. intermedia sensu lato and
L. whitmani (Marcondes et al. 1997). The haplotypes of the North-South mtDNA lineage of L. whitmani are characterized by a diagnostic, nonsynonymous G->T transversion (character 286) in the first position of the codon for the penultimate amino acid of cyt b (underlined in Fig. 2), so that serine (TCA) in the North-South haplotypes replaces the alanine (GCN; where $\mathrm{N}=\mathrm{A}, \mathrm{C}, \mathrm{G}$ or $\mathrm{T}$ ) characteristic of all other haplotypes except whit 18 from JA, which has an aspartate (GAT) in this position. 
No cyt b haplotype of L. whitmani was obviously sex-linked (Figs 2,3). This is consistent with maternal inheritance of the mitochondrial genome, which is usually true for higher eukaryotes (Avise 1994).

\section{DISCUSSION}

These studies have demonstrated that the mtDNA cyt b haplotypes of $L$. whitmani collected from Rondônia, in southwest Amazônia, belong to a monophyletic group (or clade) comprising all the haplotypes found in the rain-forest regions of this species' Brazilian range (Fig. 3). This rainforest mtDNA lineage contains a well-supported clade from the Atlantic forest zone of the northeast, including IL the type locality of $L$. whitmani, as well as the less well-supported clades from Rondônia and eastern Amazônia. The rain-forest mtDNA lineage is distinct from the North-South lineage found mainly in the drier interior of Brazil (locations TE, MA, VI), where the annual rainfall is less than $1500 \mathrm{~mm}$ (Fig.1).

Analysis of Rondônia populations was informative, demonstrating how their cyt $b$ haplotypes share character-states with haplotypes from eastern Amazônia (characters 192, 195, 288; Fig. 2) as well as with those from the northeast of Brazil (characters 201, 288). The latter two lineages now become less distinctive because of losing diagnostic character-states $(201,288)$. The cyt b haplotypes found in NAV, Amapá, surprisingly shared the state of character 287 with populations from southeast Pará, not with those from JA (Figs 1, 2), and so there is no support for the suggestion (Ready et al. 1997) that there may be a separate, widespread lineage north of the River Amazon floodplain.

Our new findings do not favour recognizing two allopatric species of $L$. whitmani in the rainforest regions of Brazil. Initially, most variation in L. whitmani seemed to be discontinuous, with a Pará form transmitting Le. shawi in silvatic habitats and a Northeast form involved in the peridomestic transmission of Le. braziliensis (Rangel et al. 1996), and perhaps even a northern Amazônia lineage transmitting Leishmania guyanensis (Ready et al. 1997). Now the mtDNA evidence points more to continuous variation between $L$. whitmani populations of the rain-forest lineage - from eastern Amazônia through Rondônia to the northeast of Brazil, or vice versa - although it should be remembered that only the terminal populations along this track are incriminated as vectors of the two genetically-divergent parasites Le. shawi and Le. braziliensis. Clinal variation of chromosomally-inherited characters in the rainforest lineage would not be surprising, based on the small, overlapping genetic distances between the weakly-supported Amazonian cyt b clades, and the grouping together of the Rondônia and Northeast clades (Fig. 3).

Part of the epidemiological interest in studying genetic variation within $L$. whitmani lies in understanding the distributions of behavioural phenotypes, such as those associated with host preferences and "loyalties" (Campbell-Lendrum et al. in press) and degrees of synanthropy or "domesticity". If there is, or has been in the recent past, a continuum of inter-breeding populations of $L$. whitmani throughout the rain-forest regions of Brazil, then the frequencies of different behavioural phenotypes might well vary in response to directional selection in any single locality (i.e. by selection of alleles conferring behaviour that is advantageous in new, man-made habitats), rather than varying with the distributions of more ancient cryptic species found in different regions and habitats. Consistent with directional selection of $L$. whitmani are the findings of studies in the ecological gradient at the southeast border of Amazônia. In a location deforested some 22 years earlier, this species was found to have Amazonian cyt b haplotypes but, atypically for this region, it was abundant peridomestically (Ready et al. 1998).

Even if L. whitmani is not composed of two or more sibling species, there might still be specific associations between clonal strains of Leishmania and genotypes of this sandfly, the frequencies of which could vary regionally or according to habitat. In particular, Le. braziliensis has a large range and shows much variation based on epidemiology and serology (Lainson et al. 1994, Shaw 1994), isoenzymes (Cupolillo et al. 1997) and RAPD-PCR typing (Ishikawa et al. 1996, in press).

\section{ACKNOWLEDGEMENTS}

To Iorlando da Rocha Barata, Maria das Graças Soares da Silva, Luís Herman Soares Gil, Luís Marcelo Aranha Camargo and José Ortiz for their help with collecting phlebotomines in Rondônia and Amapá, and to Johann Testa and Julia Bartley for their assistance in the NHM, London. This work was funded by a Wellcome Trust (London) project grant, which permitted the senior author to carry out the DNA isolation and sequencing in London.

\section{REFERENCES}

Antunes PCA, Coutinho JO 1939. Notas sobre flebotomos sul-americanos. II. Descrição de Flebotomus whitmani n.sp. e da armadura bucal de algumas espécies. Bol Biologico IV: 448-451.

Avise JC 1994. Molecular Markers, Natural History and Evolution, Chapman and Hall, New York, 511 pp.

Beard CB, Hamm DM, Collins FH 1993. The mitochondrial genome of the mosquito Anopheles gambiae: DNA sequence, genome organization, and comparisons with mitochondrial sequences of other insects. Insect Mol Biol 2: 103-124. 
Bremer K 1994. Branch support and tree stability. Cladistics 10:295-304.

Campbell-Lendrum DH, Brandão Filho SP, Ready PD, Davies CR in press. Host and/or site loyalty of Lutzomyia whitmani (Diptera: Psychodidae) in Brazil. Med Vet Entomol.

Campbell-Lendrum DH, Pinto M, Brandão Filho SP, de Souza AA, Ready PD, Davies CR in press. Experimental comparison of anthropophily amongst geographically dispersed populations of Lutzomyia whitmani. Med Vet Entomol.

Cupolillo E, Grimaldi G Jr, Momen H 1997. Genetic diversity among Leishmania (Viannia) parasites. Ann Trop Med Parasitol 91: 617-626.

Haffer J 1987. Quaternary history of tropical America, p.1-8. In TC Whitmore, GT Prance (eds), Biogeography and Quaternary History in Tropical America, Clarendon Press, Oxford.

Ishikawa EAY, Silveira FT, Shaw JJ 1996. The use of the Random-Amplified Polymorphic DNA (RAPD) technique to study the genetic polymorphism of Leishmania (Viannia) braziliensis. Mem Inst Oswaldo Cruz 91 (Suppl.): 183.

Ishikawa EAY, Silveira FT, Melo MN, Gomes R, Shaw $\mathrm{JJ}$ in press. Studies on the genetic variation of Brazilian populations of Leishmania (Viannia) braziliensis using the random amplified polymorphic DNA (RAPD) technique. Trans $\mathrm{R}$ Soc Trop Med Hyg

Lainson R, Shaw JJ, Silveira FT, de Souza AA, Braga RR, Ishikawa EAY 1994. The dermal leishmaniases of Brazil, with special reference to the eco-epidemiology of the disease in Amazônia. Mem Inst Oswaldo Cruz 89: 435-443.

Marcondes CB, Day JC, Ready PD 1997. Introgression between Lutzomyia intermedia and both Lu. neivai and Lu. whitmani, and their roles as vectors of Leishmania braziliensis. Trans $R$ Soc Trop Med Hyg 91:
725-726.

Rangel EF, Lainson R, Souza AA, Ready PD, Azevedo CR 1996. Variation between geographical populations of Lutzomyia (Nyssomyia) whitmani (Antunes \& Coutinho, 1939) sensu lato (Diptera: Psychodidae, Phlebotominae). Mem Inst Oswaldo Cruz 91: 43-50.

Ready PD, Day JC, de Souza AA, Rangel EF, Davies CR 1997. Mitochondrial DNA characterization of populations of Lutzomyia whitmani (Diptera: Psychodidae) incriminated in the peri-domestic and silvatic transmission of Leishmania species in Brazil. Bull Entom Res 87: 187-195.

Ready PD, de Souza AA, Macario Rebelo JM, Day JC, Silveira FT, Campbell-Lendrum D, Davies CR, Costa JML 1998. Phylogenetic species and domesticity of Lutzomyia whitmani at the south-east boundary of Amazonian Brazil. Trans R Soc Trop Med Hyg 92: 159-160.

Ready PD, Lainson R, Shaw JJ 1983. Leishmaniasis in Brazil: XX. Prevalence of "enzootic rodent leishmaniasis" (Leishmania mexicana amazonensis), and apparent absence of "pian bois" (Leishmania braziliensis guyanensis), in plantations of introduced tree species and in other non-climax forests in eastern Amazônia. Trans R Soc Trop Med Hyg 77: 775-785.

Shaw JJ 1994. Taxonomy of the genus Leishmania: present and future trends and their implications. Mem Inst Oswaldo Cruz 89: 471-478.

Swofford DL 1993. Phylogenetic Analysis using Parsimony (PAUP),version 3.1.1, Smithsonian Institute, Washington, USA.

Young DG, Duncan MA 1994. Guide to the identification and geographic distribution of Lutzomyia sandflies in Mexico, the West Indies, Central and South America (Diptera: Psychodidae). Mem Amer Entom Inst 54: 1-881. 
Mitochondrial Lineages of L. whitmani - EAY Ishikawa et al. 